\title{
Convergence of a Balancing Domain Decomposition by Constraints and Energy Minimization
}

\author{
Jan Mandel* Clark R. Dohrmann ${ }^{\dagger}$
}

December 2, 2002

Dedicated to Professor Ivo Marek on the occasion of his 70th birthday.

\begin{abstract}
A convergence theory is presented for a substructuring preconditioner based on constrained energy minimization concepts. The preconditioner is formulated as an Additive Schwarz method and analyzed by building on existing results for Balancing Domain Decomposition. The main result is a bound on the condition number based on inequalities involving the matrices of the preconditioner. Estimates of the usual form $C\left(1+\log ^{2}(H / h)\right)$ are obtained under the standard assumptions of substructuring theory. Computational results demonstrating the performance of method are included.
\end{abstract}

Keywords. Iterative substructuring, FETI, Balancing Domain Decomposition, NeumannNeumann, Additive Schwarz methods, non-overlapping domain decomposition methods

AMS Subject Classification. 65N55, 65F10, 65N30

\section{Introduction}

The purpose of this paper is to restate the method of Dohrmann [6] in an abstract manner, and to provide a convergence theory. The first step to this end is to define the method as an Additive Schwarz preconditioner. The main theoretical result can then be obtained by understanding the preconditioner as a Balancing Domain Decomposition type method and applying similar analysis techniques. The present analysis is focused on a two-level method for positive definite systems of equations, but we hope it will provide a solid foundation for multilevel extensions and variants for other problem types.

The Balancing Domain Decomposition (BDD) method was created by Mandel [16] by adding a coarse problem to an earlier method of Neumann-Neumann type by DeRoeck and Le Tallec [20]. The original BDD method used a multiplicative coarse correction to make sure that the residuals, which become right-hand sides of the local problems, are in the range of the substructure matrices, that is, "balanced". The method was extended to problems with jumps in coefficients between substructures by Dryja and Widlund [8] and Mandel and Brezina [18]. Le Tallec, Mandel, and

*Department of Mathematics, University of Colorado at Denver, and Department of Aerospace Engineering Sciences, University of Colorado at Boulder. Supported by the National Science Foundation under Grant DMS-0074278.

${ }^{\dagger}$ Structural Dynamics Research Department, Sandia National Laboratories, Albuquerque, New Mexico. Sandia is a multiprogram laboratory operated by Sandia Corporation, a Lockheed Martin Company, for the United States Department of Energy under Contract DE-AC04-94AL85000. 
Vidrascu [13] improved the convergence of the BDD method for plate problems by augmenting the coarse space by corner modes. Le Tallec, Mandel, and Vidrascu [14] then extended the abstract framework of BDD to allow for local substructure matrices that are no longer singular and used a coarse space consisting of corner modes only. However, the method still suffered from the loss of sparsity of the coarse matrix, which contained nonzero entries between second neighbor substructures, and the variant with nonsingular local matrices was limited to 2D.

The method of Dohrmann [6] improves on the method of [14] in many respects, yielding a coarse matrix with a favorable sparsity pattern, and extending the method to 3D in a way similar to how the FETI-DP method of Farhat et al. [9] was extended to 3D by Farhat, Lesoinne, and Pierson [10] and by Klawonn, Widlund, and Dryja [12]. Another attractive feature of the method is its potential for recursive application in a multilevel framework.

In the case of corner constraints only, the substructure spaces and the coarse space, as well as the substructure bilinear forms, are the same as in [14]. The difference is that the present method applies the coarse problem in an additive rather than multiplicative manner, which makes it possible to use a different bilinear form on the coarse space. This results in a sparser coarse matrix. The method in [14] is applicable to 2D problems only, while the present method is much more general because of the flexibility of constraints allowed. For an explicit construction of the coarse basis functions from [14], see Brenner and Sung [4]. They deal exclusively with nonconforming elements, but the algebra of the construction of the coarse basis functions is the same. The idea to add coarse degrees of freedom involving averages over edges and faces was motivated by the extension of FETI-DP to 3D in [10] and [12].

The paper is organized as follows. The essentials of Additive Schwarz methods are recalled in Section 2. We then formulate the method of [6] as an Abstract Additive Schwarz method in Section 3, identifying the assumptions needed for the construction and analysis, to allow for future applications and extensions. The focus of this paper is an algebraic analysis of the method in Section 4. The main result is a bound on the condition number based on inequalities involving matrices that make up the components of the method. In Section 5, we verify that the assumptions of our construction and analysis are satisfied for the method described in [6]. Section 6 contains a choice of the method components for finite elements and verification of the inequalities assumed by our algebraic convergence theory, yielding estimates of the usual form $C\left(1+\log ^{2}(H / h)\right)$, under assumptions usual in substructuring theory. Finally, computational results illustrating the performance of the method are presented in Section 7.

\section{Abstract Additive Schwarz preconditioner}

The Additive Schwarz preconditioner was introduced by Widlund [23] as an additive variant of the classical alternating (multiplicative) method by Schwarz [21]. The general form of the preconditioner and the condition number bound we use is due to Dryja and Widlund [8], and it is a refinement of a lemma by P.L. Lions [15]. For other related abstract bounds, see Bjørstad and Mandel [1], Mandel [17], and Matsokin and Nepomnyaschikh [19].

\subsection{Definition of Additive Schwarz preconditioner}

Let $V$ be a finite-dimensional Hilbert space and $V^{\prime}$ its dual. Consider the abstract linear problem

$$
u \in V: b(u, v)=\langle g, v\rangle, \quad \forall v \in V
$$


where $b$ is a symmetric positive definite bilinear form on $V$, and $g \in V^{\prime}$ is the right hand side. The operator form of the problem is

$$
S u=g,
$$

where the linear mapping $S: V \rightarrow V^{\prime}$ is defined by

$$
b(u, v)=(S u, v), \quad \forall u \in V, v \in V^{\prime} .
$$

An abstract Additive Schwarz preconditioner is specified by a decomposition of the space $V$ into subspaces,

$$
V=V_{0}+V_{1}+\ldots+V_{N}
$$

and by symmetric positive definite bilinear forms $b_{i}$ on $V_{i}$. The preconditioner is a linear operator

$$
M: V^{\prime} \rightarrow V, \quad M: r \mapsto u,
$$

defined by solving the problems on the subspaces and adding the results,

$$
\begin{aligned}
& u_{i} \in V_{i}, \quad b_{i}\left(u_{i}, v_{i}\right)=\left\langle r, v_{i}\right\rangle, \quad \forall v_{i} \in V_{i}, \quad i=0, \ldots, N, \\
& u=\sum_{i=0}^{N} u_{i} .
\end{aligned}
$$

\subsection{Condition number bound for an Additive Schwarz preconditioner}

Theorem 1 (Dryja and Widlund [8]) If there exist constants $C_{0}, \omega$, and a symmetric matrix $E=\left(e_{i j}\right)_{i, j=1}^{N}$ such that

$$
\begin{aligned}
\forall v \in V \quad \exists v_{i} \in V_{i}: & \sum_{i=0}^{N} b_{i}\left(v_{i}, v_{i}\right) \leq C_{0} b(v, v) \\
\forall i=0, \ldots, N \quad \forall v_{i} \in V_{i}: & b\left(v_{i}, v_{i}\right) \leq \omega b_{i}\left(v_{i}, v_{i}\right) \\
\forall i, j=1, \ldots, N \quad \forall u_{i} \in V_{i}, v_{j} \in V_{j}: & \left|b\left(u_{i}, v_{j}\right)\right| \leq e_{i j} \sqrt{b\left(u_{i}, u_{i}\right) b\left(v_{j}, v_{j}\right)}
\end{aligned}
$$

then

$$
\kappa=\frac{\lambda_{\max }(M S)}{\lambda_{\min }(M S)} \leq C_{0} \omega(1+\rho(E))
$$

where $\rho$ denotes the spectral radius.

Because $\rho(E) \leq\|E\|_{\infty}$ and we can choose $e_{i j}=1$ if $V_{i} \cap V_{j} \neq\{0\}, e_{i j}=0$ otherwise, we have the easy estimate

$$
\rho(E) \leq N_{E}=\max _{i=1, \ldots, N}\left|\left\{j \in\{1, \ldots, N\}: V_{i} \cap V_{j} \neq\{0\}\right\}\right|,
$$

i.e., $\rho(E)$ is bounded by one plus the maximal number of neighbors of a substructure.

\section{Formulation of the method}

We now present the method of Dohrmann [6] in an abstract form as an additive Schwarz method, with a suitable choice of the spaces $V_{i}$ and the bilinear forms $b_{i}$. 


\subsection{Matrix components of the method and assumptions}

We use the notation from [6], with only minor changes. The components of the method are:

- $K u=f$ is the system to be solved

- $K=\sum_{i=1}^{N} R_{i}^{T} K_{i} R_{i}$ is the global stiffness matrix size $n \times n$, assumed to be positive definite

- $K_{i}$ is the local stiffness matrix size $n_{i} \times n_{i}$ of substructure $i=1, \ldots, N$, assumed to be positive semidefinite

- $R_{i}$ is the matrix size $n_{i} \times n$ of the global to local degree of freedom (dof) mapping for substructure $i$, assumed to satisfy

$$
R_{i} R_{i}^{T}=I
$$

- $W_{i}$ is a weight matrix size $n_{i} \times n_{i}$ for substructure $i$. The weight matrices are assumed to form a decomposition of unity,

$$
\sum_{i=1}^{N} R_{i}^{T} W_{i} R_{i}=I
$$

- $R_{I i}$ is the matrix size $n_{I i} \times n_{i}$ of the restriction of vectors of dofs from substructure $i$ to its interior, i.e., to all dofs that are not on substructure boundaries; it is assumed that

$$
R_{I i} R_{I i}^{T}=I
$$

and that no interior dofs are shared by distinct substructures,

$$
i \neq j \Longrightarrow R_{I i} R_{i} R_{j}^{T}=0
$$

- $C_{i}$ is the constraint matrix size $n_{c i} \times n_{i}$ for substructure $i$. The functionals defined by the rows of $C_{i}$ are coarse dofs; we assume that the constraints do not involve interior dofs, that is,

$$
C_{i} R_{I i}^{T}=0,
$$

and that they are consistent with the weight matrices in the sense that

$$
C_{i} u_{i}=0 \Longrightarrow \forall j: C_{j} R_{j} R_{i}^{T} W_{i} u_{i}=0
$$

- $R_{c i}$ is the zero-one matrix size $n_{c i} \times n_{c}$ of the global to local coarse dof mapping for substructure $i$. The global coarse dofs are assumed to be consistent between substructures to define an interpolation in the sense that

$$
\forall v \in \mathbb{R}^{n} \exists u_{c} \forall i: C_{i} R_{i} v=R_{c i} u_{c}
$$




\subsection{The preconditioner defined as an Additive Schwarz method}

Denote the restriction from global dofs to interior dofs of the substructures by

$$
R_{I}=\left[\begin{array}{c}
R_{I 1} R_{1} \\
\vdots \\
R_{I N} R_{N}
\end{array}\right] .
$$

From (8), (10), and (11), it follows that $R_{I} R_{I}^{T}=I$. Define the space $V_{I}$ as the space of all dof vectors which may be nonzero only in the interiors of the substructures,

$$
V_{I}=\text { range } R_{I}^{T}
$$

and let $P$ denote the $K$-orthogonal projection in $\mathbb{R}^{n}$ onto $V_{I}$,

$$
P=Q K, \text { where } Q=R_{I}^{T}\left(R_{I} K R_{I}^{T}\right)^{-1} R_{I} .
$$

The method studied in this paper is the Additive Schwarz Method (2)-(3), with the spaces $V$, $V_{0}, \ldots, V_{N}$, and the bilinear forms $b, b_{0}, \ldots, b_{N}$, defined as follows. The space $V$ is defined as the $K$-orthogonal complement of $V_{I}$, and the bilinear form $b$ is given by the stiffness matrix $K$,

$$
\begin{aligned}
& V=\operatorname{range}(I-P), \\
& b(u, v)=u^{T} K v .
\end{aligned}
$$

Functions corresponding to dof vectors in $V$ are known as discrete harmonic functions. The coarse space $V_{0}$ and the coarse bilinear form $b_{0}$ are now defined by

$$
\begin{aligned}
& V_{0}=\left\{u_{0}=(I-P) \sum_{i=1}^{N} R_{i}^{T} W_{i} w_{i}: \begin{array}{c}
w_{i}^{T} K_{i} w_{i} \rightarrow \min \\
\text { s.t. } C_{i} w_{i}=R_{c i} u_{c}
\end{array}, u_{c} \in \mathbb{R}^{n_{c}}\right\}, \\
& b_{0}\left(u_{0}, u_{0}\right)=\sum_{i=1}^{N} w_{i}^{T} K_{i} w_{i} .
\end{aligned}
$$

For each substructure, there is a local space $V_{i}$ and a local bilinear form $b_{i}$, defined by

$$
\begin{aligned}
& V_{i}=\left\{u_{i}=(I-P) R_{i}^{T} W_{i} z_{i}: C_{i} z_{i}=0\right\}, \\
& b_{i}\left(u_{i}, u_{i}\right)=z_{i}^{T} K_{i} z_{i} .
\end{aligned}
$$

\subsection{Matrix form of the preconditioner}

We first need to establish some useful identities. Define $H_{i}$ as the space of dof vectors on substructure $i$ that are discrete harmonic, that is, $K_{i}$-orthogonal to all interior dofs of substructure $i$,

$$
H_{i}=\left\{u_{i}: R_{I i} K_{i} u_{i}=0\right\} .
$$

The $K_{i}$-orthogonal projection onto $H_{i}$ is

$$
P_{i}=Q_{i} K_{i}, \text { where } Q_{i}=R_{I i}^{T}\left(R_{I i} K_{i} R_{I i}^{T}\right)^{-1} R_{I i}
$$


From (8) and (11), the projection $P$ can be computed in each substructure independently,

$$
\begin{aligned}
\sum_{i=1}^{N} R_{i}^{T} P_{i} R_{i} & =\sum_{i=1}^{N} R_{i}^{T} Q_{i} K_{i} R_{i}=\sum_{i=1}^{N} R_{i}^{T} Q_{i} R_{i} R_{i}^{T} K_{i} R_{i} \\
& =\sum_{i=1}^{N} R_{i}^{T} Q_{i} R_{i} \sum_{j=1}^{N} R_{j}^{T} K_{j} R_{j} \\
& =Q K=P .
\end{aligned}
$$

Note that another way to express the fact that the projection acts in each substructure independently is that

$$
R_{j} P=P_{j} R_{j}
$$

which follows from (22), (8), and (11).

Define coarse basis functions $\Phi_{i}^{j}, j=1, \ldots, n_{c i}$, on substructure $i$ as the solutions of the problems

$$
w_{i}^{T} K_{i} w_{i} \rightarrow \min \quad \text { s.t. } C_{i} w_{i}=e_{j}
$$

where $e_{j}$ is the $j$-the column of the identity matrix, and let $\Phi_{i}=\left[\Phi_{i}^{1}, \ldots, \Phi_{i}^{n_{c i}}\right]$. Then vectors in $V_{0}$ can be written as

$$
u_{0}=(I-P) \sum_{i=1}^{N} R_{i}^{T} W_{i} w_{i}, \quad w_{i}=\Phi_{i} R_{c i} u_{c} .
$$

Because the constraints do not involve interior dofs, the vectors $w_{i}$ from (25) are discrete harmonic: we have $K_{i} w_{i}+C_{i}^{T} \lambda=0$, where $\lambda$ is a Lagrange multiplier, hence $R_{I i} K_{i} w_{i}=-R_{I i} C_{i}^{T} \lambda=0$ from (12), which gives

$$
w_{i} \in H_{i} .
$$

Finally, note that a vector $r \in \mathbb{R}^{n}$ defines a linear functional on $V$ by $\langle r, v\rangle=r^{T} v, \forall v \in V$, and from the definition of $V$, cf., (16), we have

$$
\langle r, v\rangle=r^{T} v=r^{T}(I-P) v=\left((I-P)^{T} r\right)^{T} v, \quad \forall v \in V .
$$

We are now ready to recover the preconditioner in a matrix form as in [6].

Theorem 2 Let $r \in \mathbb{R}^{n}$ be identified with a linear functional on $V$ according to (27). Then the Additive Schwarz preconditioner (2)-(3), with the spaces and bilinear forms as in (18)-(21), becomes

$$
M: r \mapsto(I-P)\left(T_{0}+\sum_{i=1}^{N} T_{i}\right)(I-P)^{T} r,
$$

where the linear mapping $T_{0}$ is defined by

$$
T_{0}: \tilde{r} \mapsto v_{0}=\sum_{i=1}^{N} R_{i}^{T} W_{i} \Phi_{i} R_{c i} K_{c c}^{-1} r_{c}
$$

with

$$
K_{c c}=\sum_{i=1}^{N}\left(\Phi_{i} R_{c i}\right)^{T} K_{i} \Phi_{i} R_{c i}, \quad r_{c}=\sum_{i=1}^{N} R_{c i}^{T} \Phi_{i}^{T} W_{i}^{T} R_{i} \tilde{r}
$$


and the linear mappings $T_{i}, i=1, \ldots, N$, are defined by

$$
T_{i}: \tilde{r} \mapsto v_{i}=R_{i}^{T} W_{i} z_{i}
$$

with $z_{i}$ being the solutions of

$$
\left[\begin{array}{cc}
K_{i} & C_{i}^{T} \\
C_{i} & 0
\end{array}\right]\left[\begin{array}{c}
z_{i} \\
\lambda
\end{array}\right]=\left[\begin{array}{c}
W_{i}^{T} R_{i} \tilde{r} \\
0
\end{array}\right]
$$

for some $\lambda$.

Remark 3 The application of $I-P$ is the static condensation correction, the map $T_{0}$ is the coarse grid correction, and $T_{i}$ constitute the substructure correction, from [6]. The multiplication of the residual by $(I-P)^{T}$ is also a static condensation correction; if $P^{T} r=0$, which will be the case, it can be omitted, and we have $r=\tilde{r}$.

Proof of Theorem 2. Let $r \in \mathbb{R}^{n}$ and $\tilde{r}=(I-P)^{T} r$. Then $\langle\tilde{r}, v\rangle=\langle r, v\rangle$ for all $v \in V$ by (27). We find the solutions of (2). Substituting into (2) for $i=0$ from (25) and using (18) and (19), we obtain

$$
\left(\sum_{i=1}^{N}\left(\Phi_{i} R_{c i}\right)^{T} K_{i} \Phi_{i} R_{c i}\right) u_{c}=\left((I-P) \sum_{i=1}^{N} R_{i}^{T} W_{i} \Phi_{i} R_{c i}\right)^{T} r
$$

which yields $u_{0}=(I-P) v_{0}$, where $v_{0}$ is given by $(29)$.

For $i=1, \ldots, N$, using (20) and (21) in (2), we have

$$
u_{i}=(I-P) v_{i}, \quad v_{i}=R_{i}^{T} W_{i} z_{i},
$$

where $z_{i}$ is found from

$$
C_{i} z_{i}=0, \quad C_{i} s_{i}=0 \Longrightarrow s_{i}^{T} K_{i} z_{i}=\left((I-P) R_{i}^{T} W_{i} s_{i}\right)^{T} r
$$

which is equivalent to

$$
z_{i} \in \operatorname{ker} C_{i}, \quad K_{i} z_{i}-W_{i}^{T} R_{i} r \perp \operatorname{ker} C_{i}
$$

which is in turn equivalent to (32).

We apply the preconditioner to the solution by conjugate gradients of the system $K x=f$ in $\mathbb{R}^{n}$, where $K$ is symmetric and positive definite. Let $x^{*}$ be the exact solution, $K x^{*}=f$ and $e^{(k)}=x^{*}-x^{(k)}$ denote the error in iteration $k$. The starting iterate $x^{(0)}$ is obtained by applying the static condensation correction to an arbitrary initial approximation $x^{(-1)}$,

$$
x^{(0)}=x^{(-1)}+Q\left(f-K x^{(-1)}\right) .
$$

Then $e^{(0)} \in V$, because, using the fact that $P$ is a projection, we have $P e^{(0)}=P(I-P) e^{(-1)}=0$. Because all search directions in conjugate gradients are also in $V$, we have all errors $e^{(k)} \in V$ for all $k \geq 0$. Hence, the residuals $r^{(k)}=f-K x^{(k)}=K e^{(k)}$ satisfy $P^{T} r^{(k)}=K Q r^{(k)}=$ $K Q K e^{(k)}=K P e^{(k)}=0$, and the first static correction in the preconditioner can be omitted. So, the preconditioned conjugate gradients run in the subspace $V$, and the well-known bound on the reduction of the error applies, cf., e.g., [11],

$$
\left\|e^{(k)}\right\|_{K} \leq 2\left(\frac{\sqrt{\kappa}-1}{\sqrt{\kappa}+1}\right)^{k}\left\|e^{(0)}\right\|_{K}
$$

where the condition number $\kappa$ is bounded from Theorem 1 .

Here and below, $\|u\|_{A}=\sqrt{u^{T} A u}$ is the norm associated with a positive definite matrix $A$. If $A$ is only positive semidefinite, we write $|u|_{A}$ instead. 


\section{Algebraic condition number bounds}

We now bound the constants in the assumptions of Theorem 1. Define the operators $D_{j i}$ by

$$
D_{j i}=R_{j}(I-P) R_{i}^{T} W_{i}=\left(I-P_{j}\right) R_{j} R_{i}^{T} W_{i},
$$

cf., (23). Note that $\sum_{i=1}^{N} D_{j i} w_{i}$ is the result of averaging the values of the vectors $w_{i}$ on the interfaces of the substructure $j$ with all of its neighbors, and extending the result into a discrete harmonic function on substructure $j$.

Theorem 4 Let the preconditioner be as in Section 3. Then the condition number satisfies

$$
\kappa \leq 5 N_{E} \max \left\{2+2 \omega_{0}, \omega_{1}\right\},
$$

where $N_{E}$ is the maximal number of neighbors of any substructure plus one, cf., (7), and

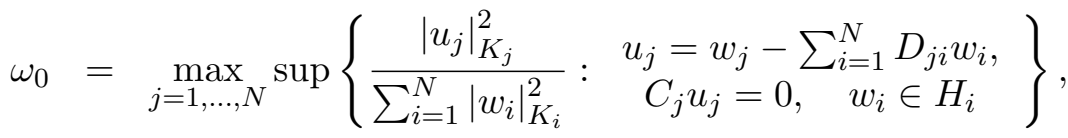

$$
\begin{aligned}
& \omega_{1}=\max _{i=1, \ldots, N} \sup \left\{\frac{\sum_{j=1}^{N}\left|D_{j i} z_{i}\right|_{K_{j}}^{2}}{\left|z_{i}\right|_{K_{i}}^{2}}: C_{i} z_{i}=0, z_{i} \in H_{i}\right\} \text {. }
\end{aligned}
$$

Remark 5 Note that the bounds $\omega_{0}$ and $\omega_{1}$ do not increase if more constraints are added to $C_{i}$.

Remark 6 The bound $\omega_{1}$ is the same as in [14, Theorem 3.4], where $b_{0}=b$, and only corner constraints were used.

Proof of Theorem 4. Let $v \in V$. Define the components in the decomposition (4) by

$$
\begin{aligned}
v_{0} & =(I-P) \sum_{i=1}^{N} R_{i}^{T} W_{i} w_{i}, \\
v_{i} & =(I-P) R_{i}^{T} W_{i} z_{i}, \quad z_{i}=R_{i} v-w_{i},
\end{aligned}
$$

where $w_{i}$ is defined by

$$
w_{i}^{T} K_{i} w_{i} \rightarrow \min \quad \text { s.t. } C_{i} w_{i}=C_{i} R_{i} v .
$$

Because $v$ is discrete harmonic, so is $R_{i} v$. From (26), $w_{i}$ is discrete harmonic, so $z_{i}$ is also discrete harmonic, $z_{i} \in H_{i}$. From (14), it follows that $C_{i} R_{i} v=R_{c i} u_{c}$ for some $u_{c}$, so $v_{0} \in V_{0}$, cf., (18). Let

$$
\alpha=b_{0}\left(v_{0}, v_{0}\right)+\sum_{i=1}^{N} b_{i}\left(v_{i}, v_{i}\right) .
$$

Then from the definitions of $b_{i}$,

$$
\alpha=\sum_{i=1}^{N} w_{i}^{T} K_{i} w_{i}+\sum_{i=1}^{N} z_{i}^{T} K_{i} z_{i}
$$

Using the definition of $z_{i}$ and the inequality $\|x+y\|^{2} \leq 2\left(\|x\|^{2}+\|y\|^{2}\right)$, we get

$$
\alpha \leq \sum_{i=1}^{N} w_{i}^{T} K_{i} w_{i}+2\left(\sum_{i=1}^{N} w_{i}^{T} K_{i} w_{i}+\sum_{i=1}^{N} v^{T} R_{i}^{T} K_{i} R_{i} v\right)
$$


and from $w_{i}^{T} K_{i} w_{i} \leq v_{i}^{T} R_{i}^{T} K_{i} R_{i} v$, we have

$$
\alpha \leq 5 \sum_{i=1}^{N} v^{T} R_{i}^{T} K_{i} R_{i} v=5 v^{T} K v
$$

Hence, (4) holds with $C_{0}=5$.

We now verify (5). For the substructure spaces, we have

$$
b\left(v_{i}, v_{i}\right)=\left\|(I-P) R_{i}^{T} W_{i} z_{i}\right\|_{K}^{2}, \quad b_{i}\left(v_{i}, v_{i}\right)=\left\|z_{i}\right\|_{K_{i}}^{2}
$$

so, for $i=1, \ldots, N,(5)$ holds with $\omega=\omega_{1}$. For the coarse space, we have

$$
\begin{aligned}
b\left(v_{0}, v_{0}\right) & =\left\|(I-P) \sum_{i=1}^{N} R_{i}^{T} W_{i} w_{i}\right\|_{K}^{2} \\
& =\sum_{j=1}^{N}\left|R_{j}(I-P) \sum_{i=1}^{N} R_{i}^{T} W_{i} w_{i}\right|_{K_{j}}^{2} \\
& \leq 2 \sum_{j=1}^{N}\left\|w_{j}\right\|_{K_{j}}^{2}+2 \sum_{j=1}^{N}\left|w_{j}-R_{j}(I-P) \sum_{i=1}^{N} R_{i}^{T} W_{i} w_{i}\right|_{K_{j}}^{2}
\end{aligned}
$$

From the decomposition of unity (9), it follows that

$$
C_{j}\left(R_{j} v-R_{j} \sum_{i=1}^{N} R_{i}^{T} W_{i} R_{i} v\right)=0 .
$$

From (34), we have $C_{j} R_{j} v=C_{j} w_{j}$. From the assumption (13), $C_{j}\left(R_{j} v-w_{j}\right)=0$ implies $C_{j} R_{j} R_{i}^{T} W_{i}\left(R_{j} v-w_{j}\right)=0$. Consequently, from (36), it follows that

$$
C_{j}\left(w_{j}-R_{j} \sum_{i=1}^{N} R_{i}^{T} W_{i} w_{i}\right)=0
$$

Further, using the assumption that the constraints do not involve interior dofs,

$$
C_{j} R_{j} P=C_{j} R_{j} Q K=C_{j} R_{j} \sum_{i=1}^{N} R_{i}^{T} R_{I i}^{T}\left(R_{I i} K_{i} R_{I i}^{T}\right)^{-1} R_{I i} K=0
$$

because $R_{j} R_{i}^{T} R_{I i}^{T}=0$ for $i \neq j$ from (11), and $C_{j} R_{j} R_{j}^{T} R_{I j}^{T}=C_{j} R_{I j}^{T}=0$ from (8) and (12). Using (38), equation (37) becomes

$$
C_{j} u_{j}=0, \text { where } u_{j}=w_{j}-R_{j}(I-P) \sum_{i=1}^{N} R_{i}^{T} W_{i} w_{i}
$$

Now (35) with the fact that $w_{i} \in W_{I}$, cf., (26), and the definition of $b_{0}$, cf., (19), gives (5) for $i=0$ with $\omega=2+2 \omega_{0}$. Finally, (6) follows from (7). 


\section{Verification of assumptions for a specific implementation}

We now present details for a specific implementation of the method and verify the assumptions of Section 3.1. Much of this section closely follows [6], but some changes were needed in order to satisfy the assumption in (13) for general unstructured meshes.

Each element of a finite element mesh is assigned to exactly one substructure. Consequently, the global stiffness matrix can be expressed in the assembled form $K=\sum_{i=1}^{N} R_{i}^{T} K_{i} R_{i}$ as assumed. In addition, the assumption in (11) follows directly from the fact that no finite element contains nodes from the interiors of two different substructures. The assumptions in (8) and (10) are satisfied because each row of $R_{i}$ and $R_{I i}$ has exactly one nonzero of unity and no column of either matrix has more than one nonzero entry.

A node is on the boundary of a substructure if it is contained in two or more substructures. Let $S_{i}$ denote the set of all nodes on the boundary of substructure $i$ and define $\mathcal{N}$ as the union of all $S_{i}$. The set $\mathcal{N}$ is expressed as the union of disjoint node subsets $\mathcal{N}_{k}$ satisfying the property

$$
s(p)=s(q) \quad \forall p, q \in \mathcal{N}_{k}
$$

where $s(p)$ is the set of all substructures containing node $p$. In words, each node of $\mathcal{N}$ is contained in exactly one node subset and all nodes in a given node subset are contained in exactly the same set of substructures. Obtaining node subsets with these properties is straightforward. Node subsets containing just a single node are called corners. Node subsets involving exactly two substructures are called faces. The remaining node subsets are called edges. For example, a cube substructure embedded in 26 adjacent cube substructures has eight corners, six faces, and twelve edges.

Each row of the constraint matrix $C_{i}$ is associated with a certain node subset $\mathcal{N}_{k} \supset S_{i}$. Thus, the assumption in (12) is satisfied because the constraints only involve dofs on substructure boundaries. Whether or not a node subset is used in the constraints is a matter of choice. For example, results are presented in Section 7 where only faces are used in $C_{i}$. In order to simplify the notation, we assume that the number of dofs per node is unity and the number constraints associated with each node subset is at most unity. Extensions for the more general case are straightforward. Let $r_{k i}$ denote the $1 \times n_{c i}$ zero-one matrix which maps rows of $C_{i}$ to the constraint associated with $\mathcal{N}_{k}$. A specific column of $r_{k i} C_{i}$ is nonzero only if it is associated with a node in $\mathcal{N}_{k}$ in which case it equals the corresponding diagonal entry of $K_{i}$. If the column is associated with a node having more than one dof, then its value equals the trace of the corresponding submatrix of $K_{i}$ for the node. Each row of $C_{i}$ is then normalized to have unity row sum.

Given $\mathcal{N}_{k} \supset S_{i}$ and $\mathcal{N}_{k} \supset S_{j}$, it follows from the construction of $C_{i}$ and (39) that

$$
r_{k i} C_{i} u_{i}=r_{k j} C_{j} u_{j} \quad \forall k
$$

In words, the constraints are consistent from substructure to substructure. Consequently,

$$
C_{i} u_{i}=0 \Longrightarrow C_{j} R_{j} R_{i}^{T} u_{i}=0 \quad \forall j
$$

Although (40) is very similar to the consistency assumption (13), the two are not equivalent for all choices of the weight matrix $W_{i}$.

The matrix $W_{i}$ in $[6]$ is given by

$$
W_{i}=\left(\operatorname{diag}\left(R_{i} K R_{i}^{T}\right)\right)^{-1} \operatorname{diag}\left(K_{i}\right)
$$

This choice of $W_{i}$ satisfies (9), but there is no reason to expect that (13) follows in general. The key to satisfying the consistency assumption is to require equality of all the diagonal entries in $W_{i}$ 
associated with a given constraint. If this is done, then one can show that (40) implies (13). To this end, define the entries of $\tilde{C}_{i}$ to be unity in the nonzero rows and columns of $C_{i}$ and zero elsewhere. The modified weight matrix is then given by

$$
\tilde{W}_{i}=\tilde{C}_{i}^{T}\left(\operatorname{diag}\left(R_{c i} K_{c c} R_{c i}^{T}\right)\right)^{-1} \operatorname{diag}\left(\Phi_{i}^{T} K_{i} \Phi_{i}\right) \tilde{C}_{i}
$$

Notice if a dof is not involved in any constraints, then the corresponding diagonal entry of $\tilde{W}_{i}$ is zero. If this is the case, then this entry is replaced by its corresponding value in (41). The modified weight matrix thus obtained satisfies the assumption in (9). Having satisfied all the assumptions of Section 3.1, it follows that Theorem 4 gives an estimate of convergence for the method of [6].

As a final note, additional corners may be needed to avoid potential singularity of the coarse problem stiffness matrix $K_{c c}$ or the coefficient matrix in (32). For example, consider an elasticity problem with two substructures connected along a shared surface. In this case there are no corners, one face, and no edges. Let $d=2,3$ denote the spatial dimension. Note that at least $3(d-1)$ constraints must be applied to each substructure to remove the possibility of rigid body motion. If one of the two substructures does not have any essential boundary conditions, then the coefficient matrix in (32) would be singular because the number of constraints associated with the face is only $d$. One method for choosing additional corners is described in [6]. Ensuring a minimal number of corners for each substructure also permits the use of software for sparse positive definite systems in the solution of (32).

\section{Condition number bounds for finite elements}

\subsection{Boundary value problem and its discretization}

Consider a self-adjoint elliptic boundary value problem

$$
\mathcal{A u}=\mathrm{g} \quad \text { in } \Omega+\text { boundary conditions on } \partial \Omega,
$$

where $\mathcal{A}$ is a self-adjoint linear elliptic operator and $\Omega$ is a bounded domain in $\mathbb{R}^{d}$. We assume that the model problem (42) is discretized by finite elements. Denote by $V_{h}(\Omega)$ the corresponding finite element space that satisfies the usual regularity and inverse properties [5], and denote by $h$ the characteristic element size. The functions in $V_{h}(\Omega)$ are assumed to satisfy the essential boundary conditions on $\partial \Omega$. As usual in substructuring theory, we assume that the domain $\Omega$ is decomposed into nonoverlapping subdomains $\Omega_{i}, i=1, \ldots, N$, which form a quasi-regular triangulation of the domain $\Omega$. Consistent with the terminology in the rest of the paper, the subdomains will be henceforth called substructures. Assume that each substructure is the union of some of the elements. The characteristic size of the substructures is denoted by $H$. Denote the space of restrictions of functions from $V_{h}(\Omega)$ to the substructures by $V_{h}\left(\Omega_{i}\right)$, and the spaces of traces by $V_{h}\left(\partial \Omega_{i}\right), i=1, \ldots, N$. For every vector of degrees of freedom $u_{i}$, denote by $\mathrm{u}_{i}=\mathcal{I}_{h} u_{i} \in V_{h}$ the corresponding finite element function. The trace of this function on substructure boundary is determined by the values of the degrees of freedom on the boundary only. Functions in $V_{h}\left(\partial \Omega_{i}\right)$ are understood to be extended to $V_{h}\left(\Omega_{i}\right)$ as discrete harmonic, and functions in $V_{h}\left(\Omega_{i}\right)$ are understood to be extended to $V_{h}(\Omega)$ by setting all degrees of freedom not associated with $\Omega_{i}$ to zero. Denote the edges (in 2D) or faces (in 3D) of the substructures by

$$
\Gamma_{i j}=\partial \Omega_{i} \cap \partial \Omega_{j}
$$

Define operators corresponding to $D_{j i}$, cf., (33), which act on finite element functions instead of vectors of dofs, by

$$
\mathrm{v}_{j}=\mathrm{D}_{j i} \mathrm{u}_{i} \Longleftrightarrow v_{j}=D_{j i} u_{i}
$$


Note that because of the convention that all functions are extended by zero, the operator $\mathrm{D}_{j i}$ is the selection of values of its argument on $\Gamma_{i j}$, multiplication by the averaging weight, and extending by zero degrees of freedom.

Let $C$ denote a generic constant independent of $h$ and $H$. The symbol $\approx$ denotes the equivalence of norms or seminorms, with constants independent of $h$ and $H$.

\subsection{An abstract estimate using a trace seminorm}

We make the assumption that there exists a trace seminorm $\left|u_{i}\right|_{\tau(\Gamma)}$, for $\Gamma \subset \partial \Omega_{i}$, such that $\left|u_{i}\right|_{\tau(\Gamma)}$ depends only on the values of $\mathrm{u}_{i}$ on $\Gamma$, and it holds that

$$
\begin{gathered}
\Gamma_{a} \subset \Gamma_{b} \subset \partial \Omega \Longrightarrow\left|\mathrm{u}_{i}\right|_{\tau\left(\Gamma_{a}\right)} \leq\left|\mathrm{u}_{i}\right|_{\tau\left(\Gamma_{b}\right)} \\
\left|u_{i}\right|_{K_{i}} \approx\left|\mathrm{u}_{i}\right|_{H^{1 / 2}\left(\partial \Omega_{i}\right)}, \quad u_{i} \in H_{i} .
\end{gathered}
$$

This will allow us to get a bound on the condition number from the growth of the trace seminorm when a function satisfying the constraints of the method on a substructure edge (or face in 3D) is multiplied by the averaging weight and extended by zero to the whole substructure boundary.

Theorem 7 Assume the problem setting from Section 6.1, that (44) holds, and that

$$
z_{i} \in H_{i}, C_{i} z_{i}=0 \Longrightarrow\left|D_{i j} z_{i}\right|_{\tau\left(\partial \Omega_{i}\right)}^{2} \leq \varpi\left|z_{i}\right|_{\tau\left(\Gamma_{i j}\right)}^{2}
$$

Then $\kappa \leq C \varpi$.

Proof. With (44), the inequalities assumed in Theorem 4 become

$$
\left|\mathrm{u}_{j}\right|_{\tau\left(\partial \Omega_{j}\right)}^{2} \leq C \omega_{0} \sum_{i: \Gamma_{i j} \neq \emptyset}\left|\mathrm{w}_{i}\right|_{\tau\left(\partial \Omega_{i}\right)}^{2}
$$

for

$$
\mathrm{u}_{j}=\mathrm{w}_{j}-\sum_{i: \Gamma_{i j} \neq \emptyset} \mathrm{D}_{j i} \mathrm{w}_{i}, \quad C_{j} u_{j}=0
$$

and

$$
\sum_{i: \Gamma_{i j} \neq \emptyset}\left|\mathrm{D}_{j i} \mathrm{z}_{i}\right|_{\tau\left(\partial \Omega_{j}\right)}^{2} \leq C \omega_{1}\left|\mathrm{z}_{i}\right|_{\tau\left(\partial \Omega_{i}\right)}^{2}, \quad \text { for } C_{j} z_{j}=0 .
$$

Consider (46). From (45), (47), (43), and the fact that the number of neighbors of a substructure is bounded, it follows that

$$
\begin{aligned}
\left|\mathrm{u}_{j}\right|_{\tau\left(\partial \Omega_{j}\right)}^{2} & =\left|\sum_{i: \Gamma_{i j} \neq \emptyset} \mathrm{D}_{j i}\left(\mathrm{w}_{j}-\mathrm{w}_{i}\right)\right|_{\tau\left(\partial \Omega_{j}\right)}^{2} \\
& \leq C \varpi \sum_{i: \Gamma_{i j} \neq \emptyset}\left|\mathrm{w}_{i}\right|_{\tau\left(\Gamma_{i j}\right)}^{2} \leq C \varpi \sum_{i: \Gamma_{i j} \neq \emptyset}\left|\mathrm{w}_{i}\right|_{\tau\left(\partial \Omega_{i}\right)}^{2}
\end{aligned}
$$

Now consider (48). Similarly as above, we have

$$
\sum_{i: \Gamma_{i j} \neq \emptyset}\left|\mathrm{D}_{j i} \mathrm{z}_{i}\right|_{\tau\left(\partial \Omega_{j}\right)}^{2} \leq C \varpi \sum_{i: \Gamma_{i j} \neq \emptyset}\left|\mathrm{z}_{i}\right|_{\tau\left(\Gamma_{i j}\right)}^{2} \leq C \varpi \sum_{i: \Gamma_{i j} \neq \emptyset}\left|\mathrm{z}_{i}\right|_{\tau\left(\partial \Omega_{i}\right)}^{2} .
$$




\subsection{Estimates for finite elements}

Using inequalities well-known from the study of substructuring methods, we are now able to obtain the usual quasi-optimal bounds on the condition number, i.e., bounds depending only weakly on $H / h$.

Suppose the weight matrices are defined by averaging between substructures using counts: $W_{i}=\operatorname{diag}\left(w_{i, j j}\right)$, where $w_{i, j j}=1 / n_{i, j j}, n_{i, j j}$ is the number of substructures that share the degree of freedom $j$ of substructure $\Omega_{i}$.

When $d=2$ or 3 and $\mathcal{A}$ is the second order scalar operator

$$
\mathcal{A} \mathrm{v}=-\sum_{i, j=1}^{d} \frac{\partial}{\partial x_{i}}\left(\alpha(x) \frac{\partial \mathrm{v}(x)}{\partial x_{j}}\right),
$$

where $0<\alpha_{0} \leq \alpha(x) \leq \alpha_{1}$ a.e. in $\Omega$ for some constants $\alpha_{0}$ and $\alpha_{1}$, a suitable trace seminorm is the Sobolev $H^{1 / 2}$ seminorm, defined by

$$
|\mathrm{u}|_{\tau(\Gamma)}^{2}=|\mathrm{u}|_{H^{1 / 2}(\Gamma)}^{2}=\int_{\Gamma} \int_{\Gamma} \frac{|\mathrm{u}(x)-\mathrm{u}(y)|^{2}}{|x-y|^{d}} d x d y .
$$

It is well known that the equivalence of seminorms (44) holds, cf., Bramble, Pasciak, and Schatz [2], Dryja [7], and Widlund [22].

Theorem 8 Suppose $d=2$, the problem (42) is of second order as in (49), discretized by standard Lagrange finite elements, and the constraint matrices are defined so that $C_{i} u_{i}$ is the vector of the values of $u_{i}$ at the corners of $\Omega_{i}$. Then $\kappa \leq C\left(1+\log ^{2}(H / h)\right)$.

Proof. From [23, Lemma 3.3], for any function v in $V_{h}\left(\Gamma_{i j}\right)$, which vanishes at the endpoints of $\Gamma_{i j}$ and is extended by zero to the whole $\partial \Omega_{j}$, it holds that

$$
|\mathrm{v}|_{H^{1 / 2}\left(\partial \Omega_{j}\right)}^{2} \leq C\left(1+\log ^{2} \frac{H}{h}\right)|\mathrm{v}|_{H^{1 / 2}\left(\Gamma_{i j}\right)}^{2},
$$

which gives (45) with $\varpi=C\left(1+\log ^{2}(H / h)\right)$.

Theorem 9 Suppose $d=3$, the problem (42) is of second order as in (49), discretized by standard Lagrange finite elements, and the constraint matrices are defined so that $C_{i} u_{i}$ is the vector of the averages of $u_{i}$ over the faces of $\Omega_{i}$. Then $\kappa \leq C\left(1+\log ^{2}(H / h)\right)$.

Proof. For $\mathrm{v} \in V_{h}\left(\partial \Omega_{i}\right)$ and $\Gamma \subset \Omega$, denote by $\mathrm{v}_{\Gamma}$ the function obtained from $\mathrm{v}$ by setting all dofs other than those associated with $\Gamma$ to zero. From [7] and [3], for any function $\mathrm{v}$ in $V_{h}\left(\Gamma_{i j}\right)$ with zero average over $\Gamma_{i j}$,

$$
\left|\mathrm{v}_{\Gamma}\right|_{H^{1 / 2}\left(\partial \Omega_{j}\right)}^{2} \leq C\left(1+\log ^{2} \frac{H}{h}\right)\left|\mathrm{v}_{i j}\right|_{H^{1 / 2}\left(\Gamma_{i j}\right)}^{2}
$$

when $\Gamma$ is $\Gamma_{i j}$ or an edge, or a corner of $\Gamma_{i j}$. This gives (45) with $\varpi=C\left(1+\log ^{2}(H / h)\right)$.

Remark 10 From Remark 5, the same estimate applies when constraints are added involving values at corners and averages over edges, as it is done in [6]. The variant of the method with face constraints only is new. This new method has a significantly smaller coarse space and works well for scalar problems, but it is not suitable for elasticity problems with displacement degrees of freedom only, because the constraints on averages of displacements over an edge (or a face in 3D) do not always eliminate all rigid body motions. Cf., computational experiments reported in Section 7. Further developments and theory for elasticity will be presented elsewhere. 
Figure 1: Illustration of a coarse basis function.

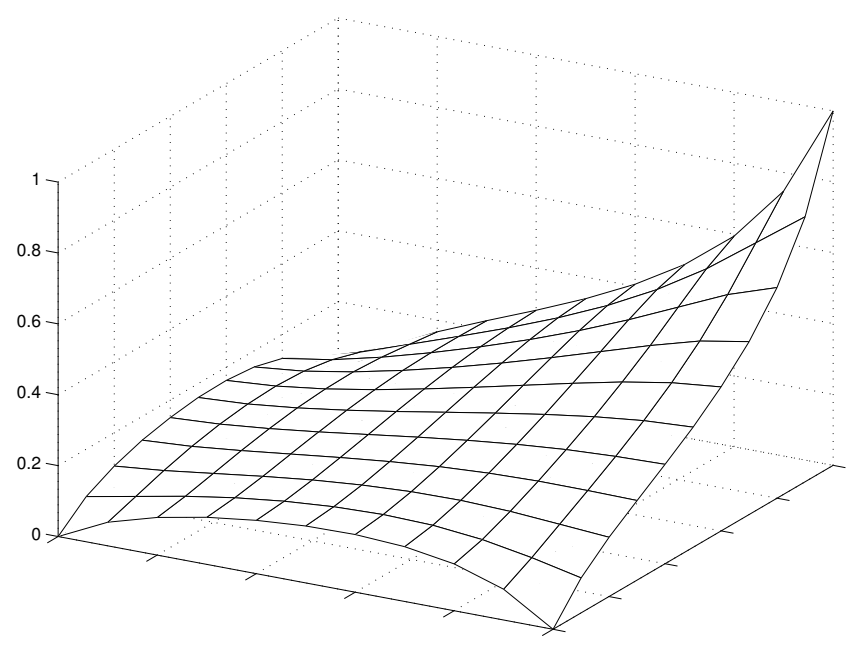
is

For the plate bending problems discretized by the HCT or DKT element, the trace seminorm

$$
|\mathrm{u}|_{\tau(\Gamma)}^{2}=|\nabla \mathrm{u}|_{H^{1 / 2}(\Gamma)}^{2},
$$

and again (44) holds, cf., [14].

Theorem 11 Suppose $d=2$, the problem (42) is plate bending, discretized by the DKT or HCT element, and the constraint matrices are defined so that $C_{i} u_{i}$ is the vector of the displacement values of $u_{i}$ at the corners of $\Omega_{i}$. Then $\kappa \leq C\left(1+\log ^{2}(H / h)\right)$

Proof. From [14], for any function v in $V_{h}\left(\Gamma_{i j}\right)$, which has zero displacement at the endpoints of $\Gamma_{i j}$ and is extended by zero dofs to the whole $\partial \Omega_{j}$, it holds that that

$$
|\nabla \mathrm{v}|_{H^{1 / 2}\left(\partial \Omega_{j}\right)}^{2} \leq C\left(1+\log ^{2} \frac{H}{h}\right)|\nabla \mathrm{v}|_{H^{1 / 2}\left(\Gamma_{i j}\right)}^{2}
$$

which gives (45) with $\varpi=C\left(1+\log ^{2}(H / h)\right)$.

\section{Computational Results}

We now present computational results for some example problems. Problems types considered in this section include the scalar Laplace equation, plate bending, and elasticity. A more comprehensive set of examples for both structured and unstructured meshes can be found in [6].

As an illustration, Figure 1 shows a coarse basis function for a second-order scalar equation discretized on a square domain, obtained by fixing its values at the corners and minimizing its energy according to (24). Summing all four basis functions leads to a constant value of unity throughout the entire domain. The ability of the coarse basis functions to reproduce the nullspace of $K_{i}$ is essential to obtaining good coarse grid corrections.

The first set of examples deals with structured meshes and ideal mesh decompositions. The purpose here is to provide numerical examples which parallel the theoretical results of Section 6 . 
Table 1: Iterations and condition number estimates for 2D problems with increasing numbers of substructures for $H / h=8$.

\begin{tabular}{|c|c|c|c|c|c|c|c|c|c|c|c|c|}
\hline & \multicolumn{4}{|c|}{ Laplace equation } & \multicolumn{3}{c|}{ plate bending } & \multicolumn{4}{c|}{ plane stress } \\
\hline & \multicolumn{2}{|c|}{ C } & \multicolumn{2}{|c|}{$\mathrm{F}$} & \multicolumn{2}{|c|}{ A } & \multicolumn{3}{|c|}{ C } & \multicolumn{3}{c|}{ C } \\
\hline$N$ & iter & $\kappa$ & iter & $\kappa$ & iter & $\kappa$ & iter & $\kappa$ & iter & $\kappa$ & iter & $\kappa$ \\
\hline 16 & 8 & 2.8 & 7 & 1.7 & 4 & 1.2 & 18 & 6.3 & 12 & 3.6 & 12 & 1.7 \\
64 & 12 & 3.1 & 8 & 1.8 & 5 & 1.3 & 23 & 6.5 & 17 & 4.8 & 22 & 70 \\
144 & 13 & 3.1 & 8 & 1.8 & 4 & 1.2 & 25 & 6.6 & 18 & 5.2 & 30 & 160 \\
256 & 13 & 3.2 & 8 & 1.8 & 4 & 1.2 & 26 & 6.6 & 19 & 5.4 & 39 & 286 \\
400 & 13 & 3.2 & 8 & 1.8 & 4 & 1.2 & 26 & 6.6 & 20 & 5.6 & 51 & 450 \\
\hline
\end{tabular}

Table 2: Results for 3D problems with increasing numbers of substructures for $H / h=8$.

\begin{tabular}{|c|c|c|c|c|c|c|c|c|c|c|}
\hline & \multicolumn{5}{|c|}{ Laplace equation } & \multicolumn{4}{c|}{ elasticity } \\
\hline & \multicolumn{2}{|c|}{ C } & \multicolumn{2}{|c|}{$\mathrm{F}$} & \multicolumn{2}{|c|}{$\mathrm{A}$} & \multicolumn{3}{|c|}{ C } & \multicolumn{2}{c|}{$\mathrm{A}$} \\
\hline$N$ & iter & $\kappa$ & iter & $\kappa$ & iter & $\kappa$ & iter & $\kappa$ & iter & $\kappa$ \\
\hline 64 & 15 & 27 & 9 & 2.0 & 6 & 1.4 & 45 & 46 & 13 & 3.6 \\
216 & 24 & 28 & 9 & 2.0 & 6 & 1.4 & 56 & 51 & 14 & 4.0 \\
512 & 34 & 28 & 10 & 2.1 & 5 & 1.4 & 59 & 54 & 14 & 4.0 \\
1000 & 36 & 29 & 10 & 2.1 & 5 & 1.4 & 62 & 55 & 14 & 4.1 \\
\hline
\end{tabular}

Consider a unit square (cube) domain with essential boundary conditions applied to the left and right sides. The domain is decomposed into $(1 / H)^{d}$ square (cube) substructures each containing $(H / h)^{d}$ quadrilateral (hexahedral) elements. Thus, the length of each substructure and element equals $H$ and $h$, respectively. For Laplace's equation, unit loads are applied to all the nodes and the diffusivity constant is set to a constant value of 1 . For plate bending, the square domain is discretized with DKT elements and unit loads are applied to all nodes in the out of plane direction. Young's modulus, Poisson's ratio, and plate thickness are set to constant values of 1, 0.3, and 0.01, respectively. The same values of Young's modulus and Poisson's ratio are used for the elasticity problems where a unit load is applied to all nodes in a coordinate direction parallel to the constrained sides. Results corresponding to the use of different node subsets in $C_{i}$ are designated by $\mathrm{C}$ for corners only, $\mathrm{F}$ for faces only, and $\mathrm{A}$ for all node subsets.

The number of preconditioned conjugate gradient (PCG) iterations needed to achieve a relative residual tolerance $\|r\|_{2} /\|f\|_{2}$ of $10^{-6}$ are shown in Tables 1 and 2 for increasing numbers of substructures. No re-orthogonalization of the PCG search directions was done for any of the results shown. Also shown in the tables are condition number estimates obtained using the connection between conjugates gradients and the Lanczos method [11]. These estimates are lower bounds of the actual condition numbers and were obtained from the extremal eigenvalues of a tridiagonal matrix of dimension equal to the number of iterations. Notice in Table 1 that condition numbers for plane stress elasticity grow quickly for case $\mathrm{F}$ as the number of substructures increases. The problem can be attributed to a coarse stiffness matrix $K_{c c}$ with nonphysical low energy modes. The problem is even worse in $3 \mathrm{D}$ where $K_{c c}$ can be singular. Thus, the use of faces only for constraints is not recommended for second-order elasticity problems.

Results for a fixed number of substructures and increasing values of $H / h$ are shown in Tables 3 and 4. Notice in Table 4 that condition numbers are much higher if only corners are used in the constraints. Similar behavior has been observed for FETI-DP [9] applied to second-order problems 
Table 3: Results for 2D problems with 16 substructures and increasing numbers of elements per substructure.

\begin{tabular}{|c|c|c|c|c|c|c|c|c|c|c|}
\hline & \multicolumn{7}{|c|}{ Laplace equation } & \multicolumn{2}{c|}{ plate bending } & \multicolumn{2}{|c|}{ plane stress } \\
\hline & \multicolumn{2}{|c|}{ C } & \multicolumn{2}{|c|}{$\mathrm{F}$} & \multicolumn{2}{|c|}{$\mathrm{A}$} & \multicolumn{2}{|c|}{$\mathrm{C}$} & \multicolumn{2}{|c|}{$\mathrm{C}$} \\
\hline$H / h$ & iter & $\kappa$ & iter & $\kappa$ & iter & $\kappa$ & iter & $\kappa$ & iter & $\kappa$ \\
\hline 4 & 7 & 2.1 & 6 & 1.3 & 4 & 1.1 & 14 & 4.2 & 10 & 2.5 \\
8 & 8 & 2.8 & 7 & 1.7 & 4 & 1.2 & 18 & 6.3 & 12 & 3.6 \\
16 & 9 & 3.7 & 7 & 2.3 & 5 & 1.4 & 22 & 8.8 & 14 & 5.1 \\
32 & 10 & 4.7 & 8 & 3.1 & 6 & 1.7 & 25 & 12 & 16 & 6.9 \\
64 & 10 & 5.9 & 9 & 4.0 & 7 & 2.0 & 29 & 15 & 18 & 9.1 \\
\hline
\end{tabular}

Table 4: Results for 3D problems with 64 substructures and increasing numbers of elements per substructure.

\begin{tabular}{|c|c|c|c|c|c|c|c|c|c|c|}
\hline & \multicolumn{9}{|c|}{ Laplace equation } & \multicolumn{4}{c|}{ elasticity } \\
\hline & \multicolumn{2}{|c|}{ C } & \multicolumn{2}{|c|}{$\mathrm{F}$} & \multicolumn{2}{|c|}{ A } & \multicolumn{3}{|c|}{ C } & \multicolumn{2}{|c|}{$\mathrm{A}$} \\
\hline$H / h$ & iter & $\kappa$ & iter & $\kappa$ & iter & $\kappa$ & iter & $\kappa$ & iter & $\kappa$ \\
\hline 4 & 10 & 8.9 & 7 & 1.5 & 4 & 1.1 & 26 & 15 & 9 & 2.0 \\
8 & 15 & 27 & 9 & 2.0 & 6 & 1.4 & 45 & 46 & 13 & 3.6 \\
12 & 23 & 51 & 10 & 2.4 & 7 & 1.7 & 58 & 84 & 16 & 4.8 \\
16 & 28 & 77 & 11 & 2.8 & 7 & 2.0 & 65 & 126 & 18 & 5.8 \\
\hline
\end{tabular}

in 3D. A related condition number bound for FETI-DP in this case can be found in [12].

Results for material property jumps aligned with substructure boundaries are shown in Tables 5 and 6. The diffusivity constant (Young's modulus) equals unity throughout the domain except in a centered square (cube) region of dimension $1 / 2$ where it equals $\sigma$. Notice that the number of iterations and condition numbers remain bounded independently of $\sigma$.

The final example deals with a sequence of meshes for a square domain with three circular cutouts. A particular mesh and mesh decomposition for this example is shown in Figure 2. The effects of increasing the number of elements per substructure are shown in Table 7 . Although these results are not as impressive as the ones in Table 3, they do show that very good results can be obtained for unstructured meshes. More complicated examples for three-dimensional elasticity problems on unstructured meshes can be found in [6].

Table 5: Results for 2D problems with material property jumps. There are 16 substructures and $H / h=6$.

\begin{tabular}{|c|c|c|c|c|c|c|c|c|c|c|}
\hline & \multicolumn{5}{|c|}{ Laplace equation } & \multicolumn{2}{c|}{ plate bending } & \multicolumn{2}{|c|}{ plane stress } \\
\hline & \multicolumn{2}{|c|}{$\mathrm{C}$} & \multicolumn{2}{|c|}{$\mathrm{F}$} & \multicolumn{2}{|c|}{$\mathrm{A}$} & \multicolumn{3}{|c|}{$\mathrm{C}$} & \multicolumn{2}{c|}{$\mathrm{C}$} \\
\hline$\sigma$ & iter & $\kappa$ & iter & $\kappa$ & iter & $\kappa$ & iter & $\kappa$ & iter & $\kappa$ \\
\hline $10^{-4}$ & 6 & 2.2 & 6 & 1.7 & 5 & 1.2 & 15 & 5.1 & 11 & 2.8 \\
$10^{-2}$ & 7 & 2.2 & 6 & 1.7 & 5 & 1.2 & 15 & 5.1 & 11 & 2.9 \\
1 & 7 & 2.5 & 6 & 1.5 & 4 & 1.2 & 16 & 5.4 & 11 & 3.1 \\
$10^{2}$ & 7 & 2.3 & 6 & 1.7 & 5 & 1.2 & 16 & 6.0 & 12 & 3.5 \\
$10^{4}$ & 7 & 2.3 & 6 & 1.7 & 5 & 1.2 & 18 & 6.1 & 12 & 3.5 \\
\hline
\end{tabular}


Table 6: Results for 3D problems with material property jumps. There are 64 substructures and $H / h=6$.

\begin{tabular}{|c|c|c|c|c|c|c|c|c|c|c|}
\hline & \multicolumn{5}{|c|}{ Laplace equation } & \multicolumn{4}{c|}{ elasticity } \\
\hline & \multicolumn{2}{|c|}{ C } & \multicolumn{2}{|c|}{$\mathrm{F}$} & \multicolumn{2}{c|}{ A } & \multicolumn{3}{c|}{ C } & \multicolumn{2}{|c|}{ A } \\
\hline$\sigma$ & iter & $\kappa$ & iter & $\kappa$ & iter & $\kappa$ & iter & $\kappa$ & iter & $\kappa$ \\
\hline $10^{-4}$ & 12 & 15 & 8 & 1.8 & 6 & 1.3 & 35 & 27 & 13 & 3.2 \\
$10^{-2}$ & 12 & 15 & 8 & 1.8 & 6 & 1.3 & 35 & 28 & 12 & 3.2 \\
1 & 12 & 17 & 8 & 1.7 & 5 & 1.3 & 37 & 30 & 11 & 2.9 \\
$10^{2}$ & 14 & 18 & 9 & 2.0 & 6 & 1.3 & 41 & 37 & 12 & 2.7 \\
$10^{4}$ & 15 & 18 & 9 & 2.0 & 6 & 1.3 & 44 & 37 & 12 & 2.7 \\
\hline
\end{tabular}

Figure 2: Unstructured mesh with 3604 elements. Substructure boundaries are indicated by thick lines.

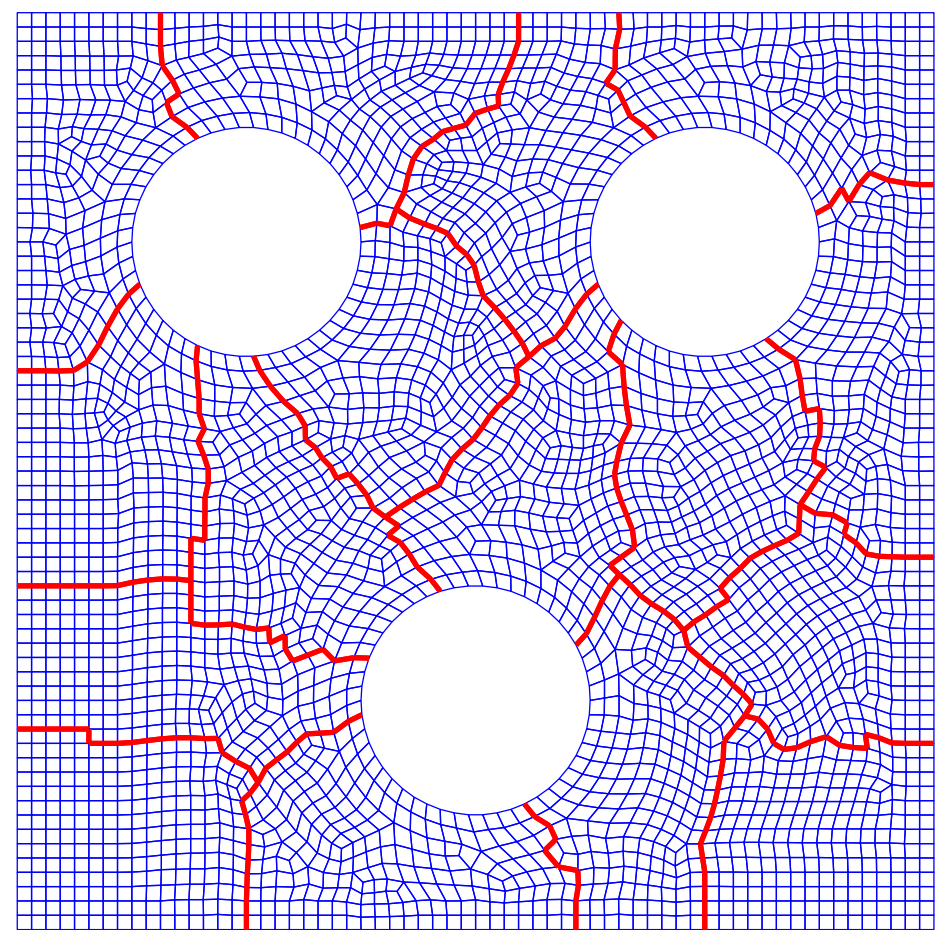

Table 7: Results for 2D unstructured mesh problems with 16 substructures. The number of elements is designated by $N_{\text {elem }}$.

\begin{tabular}{|c|c|c|c|c|c|c|c|c|c|c|}
\hline & \multicolumn{9}{|c|}{ Laplace equation } & \multicolumn{4}{c|}{ elasticity } \\
\hline & \multicolumn{2}{|c|}{ C } & \multicolumn{2}{|c|}{$\mathrm{F}$} & \multicolumn{2}{|c|}{$\mathrm{A}$} & \multicolumn{3}{c|}{ C } & \multicolumn{2}{c|}{$\mathrm{A}$} \\
\hline$N_{\text {elem }}$ & iter & $\kappa$ & iter & $\kappa$ & iter & $\kappa$ & iter & $\kappa$ & iter & $\kappa$ \\
\hline 290 & 8 & 1.8 & 9 & 1.9 & 7 & 1.8 & 12 & 2.7 & 10 & 2.5 \\
978 & 12 & 2.9 & 11 & 2.9 & 9 & 2.0 & 17 & 5.7 & 14 & 3.9 \\
3604 & 13 & 4.1 & 11 & 2.8 & 10 & 2.3 & 19 & 7.7 & 16 & 5.7 \\
12800 & 17 & 5.4 & 14 & 3.2 & 12 & 2.6 & 25 & 11 & 20 & 8.4 \\
52909 & 19 & 7.4 & 15 & 4.3 & 12 & 2.7 & 31 & 21 & 21 & 7.6 \\
\hline
\end{tabular}




\section{References}

[1] P. E. BJøRstad And J. Mandel, On the spectra of sums of orthogonal projections with applications to parallel computing, BIT, 31 (1991), pp. 76-88.

[2] J. H. Bramble, J. E. Pasciak, and A. H. Schatz, The construction of preconditioners for elliptic problems by substructuring, I, Math. Comp., 47 (1986), pp. 103-134.

[3] — The construction of preconditioners for elliptic problems by substructuring, IV, Math. Comp., 53 (1989), pp. 1-24.

[4] S. C. Brenner And L.-Y. Sung, Balancing domain decomposition for nonconforming plate elements, Numerische Mathematik, 83 (1999), pp. 25-52.

[5] P. G. Ciarlet, Basic error estimates for elliptic problems, in Handbook of Numerical Analysis, Vol. II, North-Holland, Amsterdam, 1991, pp. 17-351.

[6] C. R. Dohrmann, A preconditioner for substructuring based on constrained energy minimization, Tech. Report SAND2002-2724J, Sandia National Laboratories, Albuquerque, 2002.

[7] M. DRYJA, A method of domain decomposition for 3-D finite element problems, in First International Symposium on Domain Decomposition Methods for Partial Differential Equations, R. Glowinski, G. H. Golub, G. A. Meurant, and J. Périaux, eds., Philadelphia, PA, 1988, SIAM, pp. 43-61.

[8] M. Dryja And O. B. Widlund, Schwarz methods of Neumann-Neumann type for threedimensional elliptic finite element problems, Comm. Pure Appl. Math., 48 (1995), pp. 121-155.

[9] C. Farhat, M. Lesoinne, P. Le Tallec, K. Pierson, and D. Rixen, Feti-DP: A dualprimal unified FETI method - part I: A faster alternative to the two-level FETI method., Int. J. Numer. Meth. Engrg., 50 (2001), pp. 1523-1544.

[10] C. Farhat, M. Lesoinne, and K. Pierson, A scalable dual-primal domain decomposition method, Numer. Lin. Alg. Appl., 7 (2000), pp. 687-714.

[11] G. H. Golub And C. F. V. Loan, Matrix Computations, Johns Hopkins Univ. Press, 1989. Second Edition.

[12] A. Klawonn, O. B. Widlund, And M. Dryja, Dual-primal FETI methods for threedimensional elliptic problems with heterogeneous coefficients, SIAM J. Numer. Anal., 40 (2002), pp. 159-179 (electronic).

[13] P. Le Tallec, J. Mandel, and M. Vidrascu, Balancing domain decomposition for plates, Contemporary Mathematics, 180 (1994), pp. 515-524. Proceedings of the 7th International Symposium on Domain Decomposition Methods, Penn State, November 1993.

[14] — A Neumann-Neumann domain decomposition algorithm for solving plate and shell problems, SIAM J. Numer. Anal., 35 (1998), pp. 836-867.

[15] P.-L. Lions, On the Schwarz alternating method. I., in First International Symposium on Domain Decomposition Methods for Partial Differential Equations, R. Glowinski, G. H. Golub, G. A. Meurant, and J. Périaux, eds., Philadelphia, PA, 1988, SIAM, pp. 1-42. 
[16] J. Mandel, Balancing domain decomposition, Comm. Numer. Meth. Engrg., 9 (1993), pp. 233-241.

[17] - Hybrid domain decomposition with unstructured subdomains, Contemporary Mathematics, 157 (1994), pp. 103-112. Proceedings of the 6th International Symposium on Domain Decomposition Methods, Como, Italy, 1992.

[18] J. Mandel And M. Brezina, Balancing domain decomposition for problems with large jumps in coefficients, Math.Comp., 65 (1996), pp. 1387-1401.

[19] A. M. Matsokin and S. V. Nepomnyaschikh, A Schwarz alternating method in a subspace, Soviet Mathematics, 29(10) (1985), pp. 78-84.

[20] Y.-H. D. Roeck And P. L. Tallec, Analysis and test of a local domain decomposition preconditioner, in Proceedings of the Fourth International Symposium on Domain Decomposition Methods for Partial Differential Equations, R. Glowinski, Y. Kuznetsov, G. Meurant, J. Périaux, and O. Widlund, eds., SIAM, 1991, pp. 112-128.

[21] H. A. Schwarz, Über einen Grenzübergang durch alternierendes Verfahren, Vierteljahrsschrift der Naturforschenden Gesellschaft in Zürich, 15 (1870), pp. 272-286.

[22] O. B. WidLund, An extension theorem for finite element spaces with three applications, in Numerical Techniques in Continuum Mechanics, W. Hackbusch and K. Witsch, eds., Braunschweig/Wiesbaden, 1987, Notes on Numerical Fluid Mechanics, v. 16, Friedr. Vieweg und Sohn, pp. 110-122. Proceedings of the Second GAMM-Seminar, Kiel, January, 1986.

[23] — Iterative substructuring methods: Algorithms and theory for elliptic problems in the plane, in First International Symposium on Domain Decomposition Methods for Partial Differential Equations, R. Glowinski, G. H. Golub, G. A. Meurant, and J. Périaux, eds., Philadelphia, PA, 1988, SIAM. 